\title{
Aspects of gut development
}

\author{
BY CHARLES SHAW-SMITH ${ }^{1 *}$ AND NICHOLAS A. WRIGHT2† \\ ${ }^{1}$ Department of Gastroenterology and ${ }^{2}$ Department of Histopathology, Royal Postgraduate \\ Medical School, Hammersmith Hospital, London W12 ONN
}

\section{DEVELOPMENT OF THE GASTROINTESTINAL (GI) TRACT}

The present review examines the development of the GI tract in three different species: Drosophila, mouse and man. It makes no attempt to be comprehensive, focusing instead on those areas in which greatest progress has been made in recent years. Inevitably, as in other areas, the application of techniques in cell and molecular biology has led to the most striking advances, and in particular the use of in situ hybridization has transformed the study of developmental biology, to the extent that virtually no work is now reported which does not employ the technique in some form. It is easy to see why: developmental biology has become, at least for the time being, the study of growth factors and their regulation, and it is not enough to know merely that they are expressed in a particular tissue, a knowledge of where they are expressed in that tissue is as important.

Whilst acknowledging that the GI tract contains solid as well as hollow organs, and extends fully from mouth to anus, the present review will focus on the intestine, particularly the small intestine. It is probably the best studied organ in the GI tract from a developmental viewpoint and our understanding of its ontogeny is well in advance of that of other parts of the GI tract. The organization of the intestine in terms of its crypt-villus structure, regional differences in development, and high cell turnover make it an attractive model for the study of development, and this has been seized upon by many investigators in recent years. In Drosophila, recent work has shed light on the earliest phase of gut development, as endoderm differentiates under the influence of mesoderm. In the mouse, progress has been made in our understanding of the generation of the crypt-villus unit, and mechanisms of cytodifferentiation of gut epithelium have been elucidated. In man, the focus is of necessity even narrower.

\section{DROSOPHILA}

\section{Homeotic complex}

A major finding of recent years has been the characterization of a group of genes whose products serve to specify positional identity throughout the longitudinal axis of the Drosophila embyro. This group, termed the homeotic complex, has highly conserved counterparts in higher organisms including mouse and man (McGinnis \& Krumlauf, 1992). Its members, which include the antennapedia and ultrabithorax genes, are of interest to students of gut development because they may have a role at the earliest stage of gut ontogeny, in specifying regional differences in the early developing GI tract, and possibly also in maintaining these differences in those tissues in the adult which have a high rate of cell turnover. The products of these genes influence programmes of

* Present address: The Sanger Centre, Hinxton Hall, Hinxton, Cambridge CB10 1RQ.

$\dagger$ For reprints. 
differentiation, and their profound effects on the morphology of the developing embyro are thought to be mediated through the coordinated control of many genes at the level of transcription. Expression domains for these genes within the developing embyro are tightly regulated, and in many cases have been shown to follow simple rules: anteriorposterior gradient for maternal genes, parasegmental boundaries for homeotic genes, stripes for pair-rule genes, and so on. The defining feature of this gene family is the homeodomain, a sixty amino acid helix-loop-helix motif which contains the most highly conserved residues and which is directly involved in DNA binding, recognizing a core TAAT motif in double-stranded DNA (Gehring et al. 1994).

In early Drosophila development, two primordial ectodermal invaginations from anterior and posterior poles of the embyro grow towards each other and fuse to form a continuous tube which will develop into the foregut and mid-gut. This tube has a mesodermal and endodermal cell layer, the forebears of the gut epithelium and mesenchyme. The foregut gives rise to the mouth, buccal cavity, pharynx and oesophagus. In the mid-gut, three constrictions occur to divide it up into four morphologicallydistinct chambers which correspond to segments within the ectodermal layer of the developing embyro (Reuter et al. 1990).

\section{Homeotic genes and early gut development}

Expression domains of members of the homeotic complex have been defined for visceral mesoderm in Drosophila (Tremml \& Bienz, 1989). Antp, $U b x$ and $a b d-A$ are all expressed in adjacent parasegmental domains, the boundaries of which depend on regulatory interactions between these genes. This pattern of homeotic gene expression is not observed in the overlying endoderm. Instead, a single homeotic gene, lab, is expressed in the latter stages of mid-gut development within the endodermal cell layer. It seemed a reasonable hypothesis that induction of $l a b$ might in some way be under the control of the underlying segmented mesoderm through the medium of a diffusible factor. Previous work (St Johnson \& Gelbart, 1987) had demonstrated that expression domains coincided for $U b x$ and decapentaplegic (dpp), the latter encoding a protein sharing homology with the TGF-b gene family, and it was shown (Immergluck et al.

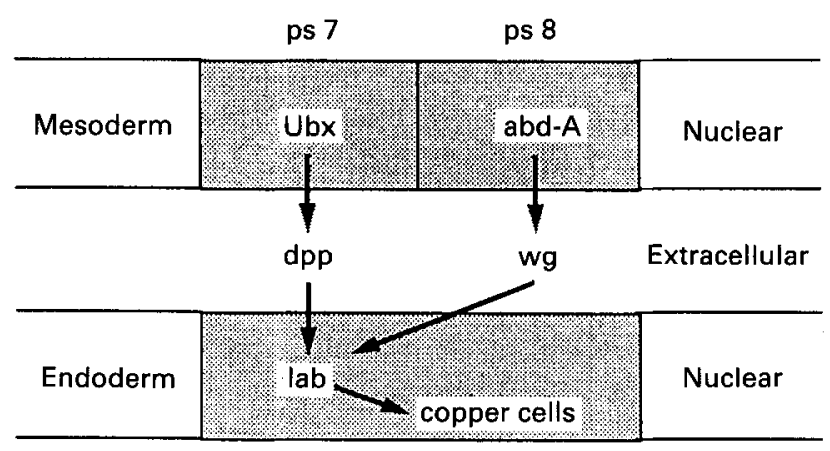

Fig. 1. Homeotic cascade in differentiation of copper cells in the Drosophila intestinal epithelium. Four different genes, $U b x, a b d-A, D p p$, and $w g$ control expression of $l a b$ in the developing endoderm. Differentiation of copper cells is in turn dependent on lab expression. For simplicity, negative interactions are not shown. (Based on Immergluck et al. 1990; Hoppler \& Bienz, 1994.) 
1990; Reuter et al. 1990) that expression of $d p p$ is abolished in the absence of $U b x$. Similar observations were made for Antp, which is expressed one parasegment down from $U b x$, and $w g$, a relative of the mammalian proto-oncogene int-1. It was possible to show also that correct expression of $l a b$ was dependent on normal function of all four of these genes, with a clear hierarchy (Fig. 1). The interpretation of these findings is that positional information is supplied by a segmented structure (mesoderm) to a nonsegmented structure (endoderm) early in development, by medium of diffusible factors, under the control of homeotic genes.

It is possible that longitudinal gradients of expression of genes such as lab may play a determinative role in the genesis of specialized tissues longitudinally along the GI tract, and evidence is emerging for a transcriptional hierarchy leading to the terminally differentiated state (Kuo et al. 1992). Hoppler \& Bienz (1994) showed that expression of $l a b$ is strictly required for formation of a highly-specialized cell type within the larval mid-gut, the copper cell, adding the weight of experimental observation to this concept.

\section{MOUSE}

Manipulation of embryonic stem cells, transgenesis, and molecular techniques for studying the control of gene expression have all contributed to a recent explosion of knowledge. This part of the review examines development of the crypt-villus unit, and control of cytodifferentiation in the murine small intestine.

\section{Early gut development}

Little information to compare with work in Drosophila concerning the earliest stages of mouse gut development is available, although comparable expression domains for genes of the homeotic complex have been established (McGinnis \& Krumlauf, 1992). Nonetheless, approaches to the study of early gut organogenesis in the mouse are beginning to be made. One novel approach exploited the observation that the zinc-finger transcription factor HNF-4 is expressed in primary endoderm in the early mouse embryo, and in fact is a marker for future endoderm development in the implanting mouse blastocyst, being later observed in pancreas, stomach and intestine (Duncan et al. 1994). Targeted disruption of the HNF-4 gene in embryonic stem cells was lethal in HNF-4/-homozygotes, with no embryos surviving beyond embryonic day (E) 11. Of particular interest, cell death was evident in the embryonic ectoderm at E 6.5 , when this tissue normally initiates gastrulation, but so little embryonic tissue of any kind developed in these homozygotes that it was impossible to draw any conclusions about later effects on gut development. The heterozygotes appeared normal (Chen et al. 1994).

In normal development, mouse fore- and hindgut first appear from endoderm on $\mathrm{E} 7$, and are followed by mid-gut $1 \mathrm{~d}$ later. Proliferation of the nascent epithelium takes place after $\mathrm{E} 10$. Between $\mathrm{E} 15$ and $\mathrm{E} 18$, a proximal to distal wave of cytodifferentiation takes place to convert the lumen surface to a simple columnar epithelium, and villi become apparent towards late gestation due to upward growth of mesenchyme at the core of each villus, and at this stage intestine-specific gene expression occurs. Crypts develop from the flat sheet of inter-villus epithelium just after birth, and between the second and third week, their number increases dramatically (Hermiston et al. 1994). 


\section{The crypt-villus unit}

Using a novel chimeric model, Schmidt et al. (1988) investigated the process of crypt formation in more detail, using mouse chimeras which contained a visual mosaic marker based on the presence in one mouse strain (B6) but not another (SWR) of binding sites for the lectin, Dolichos biflorus agglutinin (DBA). Chimeras were obtained by aggregation of four to eight cell embryos, and the chimeric animals analysed at postnatal days (P) 2, 6, 10 and 14. Within the intestine, patches of staining indicated DBA-positive crypts. At the boundaries of these patches, chimeric striped crypts were observed indicating a mixed clonal origin for that crypt. From P 2 to P 14, the number of mixed crypts expressed as a percentage of crypts at patch boundaries fell from 50 to approximately zero, indicating a process in which crypt monoclonality is gradually established in early postnatal life.

It is currently believed that the stem cell, which is the progenitor of every epithelial cell within a given crypt, is also the precursor of each of the three other cell types within the crypt, the enteroendocrine, Paneth and goblet cells. Using a mouse model which exhibited mosaicism for a transgene consisting of the liver fatty acid-binding protein (L-FABP)1 linked to a reporter gene (human growth hormone; hGH), Roth et al. (1992) showed that, in 'striped' villi originating from crypts of hGH-positive and hGH-negative type, enteroendocrine cells had a chimeric origin different from the epithelial cells within the same crypt in fewer than $0.1 \%$ of cases. This precise overlap is consistent with a common stem cell origin. Direct evidence of this kind does not exist for goblet and Paneth cells. Whether each stem cell gives rise to several daughter stem cells for each crypt cell type, or whether there is a single stem cell for the entire crypt population is still not known with certainty.

\section{Intestinal isografts}

An important question with regard to the process of gut development has been to determine to what extent the differentiation of the gut epithelium is genetically programmed, and to what extent it is determined by factors within the gut lumen. An experimental model which examines this issue directly involves the transplantation of segments of fetal small intestine into the subcutaneous tissues of young adult nude mice (Rubin et al. 1992). Pregnant mice at E 15 or E 16 were killed, and the embryos dissected to give two intestinal 'halves', proximal (duodenum and jejunum) and distal (ileum). Each part, with the ends clipped, was implanted in the subcutaneous fascia of a young adult nude male mouse (CBY/B6). At 4-6 weeks after engraftment, each intestinal segment was removed and examined histologically and by immunocytochemistry. They showed that intestinal fatty acid-binding protein (I-FABP) and L-FABP, and a variety of peptides expressed in enteroendocrine cells, showed an appropriate pattern of regionspecific differentiation. Whether or not, as Rubin et al. (1992) discuss, this spatial information is irreversibly imprinted at a discrete period in development, or whether it requires continuous expression of a particular 'control gene', is a separate question and one that arguably cannot be answered using information currently available. Although transcriptional activation of L-FABP does not occur in the fetal intestine until E 17 in the jejunum, and in the ileum 2 d later (see p. 523 and Rubin, 1992), the nature of the positional information which the intestine uses to implement this is currently a matter for speculation, and in any case may well form part of a hierarchy, as we have seen with 
Drosophila. The question of imprinting or continuous input will need to be answered for each level of this presumptive hierarchy for a full understanding to be gained. The effects of hormones and diet on intestinal maturation are discussed later (p. 525).

\section{Encoding regional specialization in the mouse gut}

Rapid progress has been made recently in establishing the mechanisms by which positional and temporal information is encoded with respect to regulation of intestinal epithelial gene expression. Nucleotide sequences of coding and regulatory regions for many intestine-specific genes have been determined. As with other eukaryotic genes, a reasonable hypothesis was that their expression is regulated at the transcriptional level, and is under the control of regions within promoter and enhancer sequences in the 5 -flanking untranscribed part of the gene. The impetus for these studies was increased by observations suggesting that the appearance of membrane-transport proteins was under transcriptional control, and was preceded by a short interval by the appearance of the appropriate mRNA (Rubin, 1992). Taking as a model two genes with expression in the adult mouse preferentially at the proximal (L-FABP) and distal (I-FABP) ends of the small intestine, it was first of all shown by in situ hybridization that transcription of L-FABP is activated in fetal jejunum between $\mathrm{E} 17$ and $\mathrm{E} 18$, and proceeds distally along the horizontal length of the gut in 'wave-like' fashion, appearing in the ileum several days later. L-FABP is not expressed in the ileum of the adult mouse, and it is clear, therefore, that both temporally- and spatially-operating factors are at work in controlling the expression of this gene. I-FABP is first detected in total intestinal RNA at E 18, and is not detected in proximal small intestine at any time during postnatal development. In contrast to L-FABP, immunocytochemical studies reveal a distal to proximal wave of activation. At P 7, the expression domain of I-FABP suddenly begins to shrink, with the effect that by $P 11$, it is confined to the caecum and a small part of distal ileum only. Following this the domain widens again to its adult pattern (Crossman et al. 1994).

\section{Promoter analysis}

In a series of promoter-mapping experiments, it has been demonstrated that upstream elements of the I-FABP gene appear to play a major role in coordinating this pattern of expression (Crossman et al. 1994). The mouse I-FABP gene with about $1 \mathrm{~kb}$ of its promoter was sequenced and a series of successive deletions of the I-FABP 5' non-transcribed domain were made by restriction enzymes. Transgenic mice were generated using these promoter fragments linked to hGH which serves as a readilyidentifiable reporter. Dramatically different temporal and spatial patterns of expression as a function of the size of the promoter fragment were seen, allowing a functional map of the I-FABP to be constructed. An interesting feature is that the appropriate information for positional expression is located in a remarkably compact sequence of 145 base pairs immediately upstream of the transcription start site. This construct in general allowed over-expression of the reporter gene, whilst the addition of elements from further upstream seemed to suppress activation to bring it more into line with the pattern seen with the native gene. Although a somewhat reductionist approach, it nonetheless demonstrates convincingly that elements for controlling expression of a gene can be separated entirely from the coding sequence of the gene itself, which can be replaced by another gene foreign to the organism. 


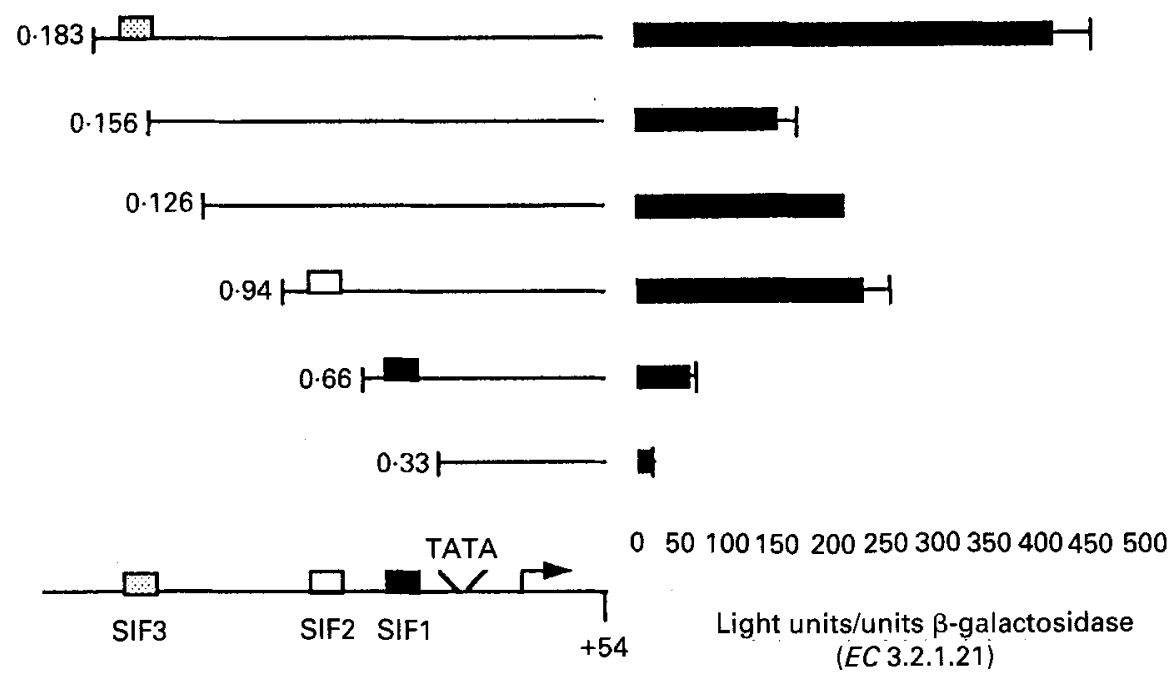

Fig. 2. Functional assessment of sucrase-isomaltase (EC 3.2.1.10; 3.2.1.48; SI) promoter. Constructs of varying length linked to the luciferase (EC 1.14.14.3) reporter gene were transfected into Caco-2 cells and the efficiency of transcription assessed. SI footprints 1-3 all appear to have functional importance. For details, see text. (Based on Traber et al. 1992.)

Work on another gene, sucrase-isomaltase (EC 3.2.1.10, 3.2.1.48; SI), a membrane disaccharidase found exclusively in small-intestinal enterocytes, and preferentially expressed in mid-small intestine, has gone further in characterizing more precisely which elements within the $5^{\prime}$ non-transcribed regions of intestinal genes are important in regulating expression. The SI gene, including $3.6 \mathrm{~kb}$ of its promoter, has been sequenced in the mouse (Wu et al. 1992). Transient transfection analysis provides a more immediate assay system than transgenesis for promoter mapping. The promoter fragment is again linked to a reporter gene in combination with a strong viral enhancer, and this construct is transfected into a cell line in culture. The most useful results are obtained by choosing a cell-line phenotypically as close as possible to small intestine, and Caco-2 cells from a colon carcinoma cell line which has features of small-intestinal differentiation give best reporter gene expression, presumably because they contain a sufficient number of smallintestinal transcription factors to activate transcription in the construct. Results obtained from DNAse I (EC 3.1.21.1) footprinting experiments made it possible to focus attention on three short nucleotide sequences within about 180 base pairs of the transcription start site. Nuclear proteins extracted from the Caco-2 cell line were shown, using the footprinting technique, to bind to three specific sites within the SI promoter (Fig. 2). Sequential removal of these sites led to a reduction in efficiency of transcription in the transient transfection assay, indicating a positive regulatory role (Traber et al. 1992).

In an extension of this work, multimers of one of the footprints, SIF1, were used to screen an adult mouse jejunum cDNA expression library. This enabled a clone to be identified, the gene product of which had the same pattern of binding to SIF1 as the crude nuclear extract (Suh et al. 1994). Sequencing of this clone revealed it to be identical in all residues but one to mouse $c d x-2$, a homeodomain protein with a counterpart in Drosophila called caudal (Duprey et al. 1988), which is related to Antp but which maps 
outside the homeotic complex. This is the first example of a direct role for a homeodomain protein in regulation of intestinal gene expression in the mouse. There are, however, several examples of homeodomain transcription factors whose expression appears not only to be confined to small-intestinal epithelium, but also to show regional variation along the longitudinal axis of the small intestine (James \& Kazenwadel, 1991; Hu et al. 1993; James et al. 1994).

\section{Influence of hormones}

There is convincing evidence from work done chiefly in the rat that these developmental programmes may be influenced by hormonal changes (Henning, 1981), and the best-studied example is the effect of glucocorticoids on membrane disaccharidase expression. Exogenous steroid administration to the 10 -d-old rat leads to precocious appearance of sucrase-isomaltase mRNA and protein, normal timing of appearance being $18 \mathrm{~d}$ (Leeper \& Henning, 1990). The interval between steroid administration and appearance of mRNA was $24 \mathrm{~h}$, leaving room for speculation on where the hormone is acting, and what other factors may be required.

\section{MAN}

Necessarily, much less information is available on the subject of human GI tract development. This section focuses on recent work on lactose intolerance, which has shed light on the ontogeny of the lactase-phlorizin hydrolase $(E C 3.2 .1 .108,3.2 .1 .62 ; \mathrm{LPH})$ enzyme in human GI tract development. As far as extending the previously described studies in Drosophila and the mouse is concerned, very little work has been done, and it is difficult to see how, ethically, it ever could be. Researchers will have to content themselves as before with the interpretation of whatever biopsy specimens are available, and the observation of the effects of genetic mutation, and the elucidation of their mechanisms.

LPH is a composite enzyme with two catalytic activities, lactase and phlorizin hydrolase. It is located in the brush-border membrane of small-intestinal epithelial cells and is responsible for hydrolysis of lactose to glucose and galactose. Its activity is high in newborn and suckling mammals, but there is a decline in the post-weaning phase with low levels in adolescents and adults of all species so far examined. Humans are not an exception to this rule, although persistence of lactase in certain human populations initially led to the belief that they were. Two distinct phenotypes are recognized, lactase persistence and lactase restriction. The genetics of this polymorphism were established in family studies in the 1970s (Sahi et al. 1973), and it was shown that, in Northern European populations, lactase persistence shows an autosomal dominant pattern of inheritance. One hypothesis for the establishment of the polymorphism is that lactase persistence may have increased by natural selection in some African populations that became dependent on milk consumption after the domestication of milking animals (Flatz, 1992), but there is as yet no convincing explanation for predominance of lactase persistence in Central and North West Europe.

In an attempt to elucidate the molecular genetics of the condition, the human LPH gene was cloned and sequenced, and comparisons made of the coding regions and intron boundaries in three human subjects with lactase persistence and one with lactase 
restriction. No sequence differences were found. Arguably, this might be regarded as an expected finding, if the change in lactase expression is seen as a polymorphism not of the gene itself but rather of the switch mechanism that turns off lactase expression shortly after weaning. There is good evidence that this switch operates at the level of gene transcription (Lloyd et al. 1992). Logically, therefore, attention has turned to the lactase promoter. In transgenic mice, the pig lactase promoter was able to down-regulate expression of a reporter gene with a pattern similar to that seen with lactase itself (Troelsen et al. 1994). In human subjects, analysis of the lactase promoter did not reveal any difference between those with lactase persistence and those with lactase restriction. (Lloyd et al. 1992). Further analysis of the lactase promoter (Troelsen, 1994) revealed a short sequence 40 base pairs upstream of the transcriptional start site which bound a nuclear factor designated NF-LPH1. The post-weaning fall in LPH levels is paralleled by a significant reduction in NF-LPH1 levels, the implication being that this nuclear protein is involved in the post-weaning down-regulation of lactase by interacting with a specific element in the lactase promoter. There are many objections to this hypothesis: high levels of lactase mRNA are detectable in small-intestinal biopsies of some patients with 'lactase restriction', indicating that post-translational regulation may be important, and there are also clear examples of mosaicism within individual villi, which are unexplained (Maiuri et al. 1994).

\section{CONCLUSION}

Where might this lead? A better understanding of stem cell origins and cell cycle control within the intestinal crypt should provide insights into the mechanisms of carcinogenesis within the GI tract. A coupling of advances in gene therapy with increased knowledge of the mechanisms of intestinal epithelial gene regulation could theoretically lead to new therapeutic avenues in specific disease states (specific enzyme deficiency, short-bowel syndrome), but this is a long way off.

C.S.-S. is a Wellcome Clinical Research Fellow. The authors thank Tristan Robbie for assistance in preparing the figures.

\section{REFERENCES}

Chen, W. S., Manova, K., Weinstein, D. C., Duncan, S. A., Plump, A. S., Prezioso, V. R., Bachvarova, R. F. \& Darnell, J. E. Jr (1994). Disruption of the HNF-4 gene, expressed in visceral endoderm, leads to cell death in embryonic ectoderm and impaired gastrulation of mouse embyros. Genes and Development $\mathbf{8}$, 2466-2477.

Crossman, M. W., Hauft, S. M. \& Gordon, J. I. (1994). The mouse ileal lipid-binding protein gene: a model for studying axial patterning during gut morphogenesis. Journal of Cell Biology 126, 1547-1564.

Duncan, S. A., Manova, K., Chen, W. S., Hoodless, P., Weinstein, D. C., Bachvarova, R. F. \& Darnell, J. E. Jr (1994). Expression of transcription factor HNF-4 in the extraembryonic endoderm, gut, and nephrogenic tissue of the developing mouse embryo: HNF-4 is a marker for primary endoderm in the implanting blastocyst. Proceedings of the National Academy of Sciences, USA 91, 7598-7602.

Duprey, P., Chowdhury, K., Dressler, G. R., Balling, R., Simon, D., Guenet, J. L. \& Gruss, P. (1988). A mouse gene homologous to the Drosophila gene caudal is expressed in epithelial cells from the embryonic intestine. Genes and Development 2, 1647-1654.

Flatz, G. (1992). Lactase deficiency: biologic and medical aspects of the polymorphism. In The Genetic Basis of Common Disease, chapt. 15 [R. A. King, J. I. Rotter and A. G. Motulsky, editors]. London: Oxford University Press.

Gehring, W. J., Affolter, M. \& Burglin, T. (1994). Homeodomain proteins. Annual Review of Biochemistry $63,487-526$. 
Henning, S. J. (1981). Postnatal development: coordination of feeding, digestion, and metabolism. American Journal of Physiology 241, G199-G214.

Hermiston, M. L., Simon, T. C., Crossman, M. W. \& Gordon, J. I. (1994). Model systems for studying cell fate specification and differentiation in the gut epithelium. In Physiology of the Gastrointestinal Tract, 3rd ed., chapt. 12 [L. R. Johnson, editor]. New York: Raven Press.

Hoppler, S. \& Bienz, M. (1994). Specification of a single cell type by a Drosophila homeotic gene. Cell 76, 689-702.

Hu, Y., Kazenwadel, J. \& James, R. (1993). Isolation and characterization of the murine homeobox gene $\mathrm{Cdx}-1$. Regulation of expression in intestinal epithelial cells. Journal of Biological Chemistry 268, 27214-27225.

Immergluck, K., Lawrence, P. A. \& Bienz, M. (1990). Induction across germ layers in Drosophila mediated by a genetic cascade. Cell 62, 261-268.

James, R., Erber, T. \& Kazenwadel, J. (1994). The structure of the murine homeobox gene cdx-2. Expression in embryonic and adult intestinal epithelium. Journal of Biological Chemistry 269, 15229-15237.

James, R. \& Kazenwadel, J. (1991). Homeobox gene expression in the intestinal epithelium of adult mice. Journal of Biological Chemistry 266, 3246-3251.

Kuo, C. J., Conley, P. B., Chen, L., Sladek, F. M., Darnell, J. E. Jr \& Crabtree, G. R. (1992). A transcriptional hierarchy involved in mammalian cell-type specification. Nature 355, 457-461.

Leeper, L. L. \& Henning, S. J. (1990). Development and tissue distribution of sucrase-isomaltase mRNA in rats. American Journal of Physiology 258, G52-G58.

Lloyd, M., Mevissen, G., Fischer, M., Olsen, W., Goodspeed, D., Genini, M., Boll, W., Semenza, G. \& Mantei, N. (1992). Regulation of intestinal lactase in adult hypolactasia. Journal of Clinical Investigation 89, 524-529.

McGinnis, W. \& Krumlauf, R. (1992). Homeobox genes and axial patterning. Cell 68, 283-302.

Maiuri, L., Rossi, M., Raia, V., Garipoli, V., Hughes, L. A., Swallow, D., Noren, O., Sjostrom, H. \& Auricchio, S. (1994). Mosaic regulation of lactase in human adult-type hypolactasia. Gastroenterology 107, $54-60$.

Reuter, R., Panganiban, G. E., Hoffmann, F. M. \& Scott, M. P. (1990). Homeotic genes regulate the spatial expression of putative growth factors in the visceral mesoderm of Drosophila embryos. Development 110, 1031-1040.

Roth, K. A., Cohn, S. M., Rubin, D. C., Trahair, J. F., Neutra, M. R. \& Gordon, J. I. (1992). Regulation of gene expression in gastric epithelial cell populations of fetal, neonatal, and adult transgenic mice. American Journal of Physiology 263, G186-G197.

Rubin, D. C. (1992). Spatial analysis of transcriptional activation in fetal rat jejunal and ileal gut epithelium. American Journal of Physiology 263, G853-G863.

Rubin, D. C., Swietlicki, E., Roth, K. A. \& Gordon, J. I. (1992). Use of fetal intestinal isografts from normal and transgenic mice to study the programming of positional information along the duodenal-to-colonic axis. Journal of Biological Chemistry 267, 15122-15133.

Sahi, T., Isokoski, M., Jussila, J., Launiala, K. \& Pyorala, K. (1973). Recessive inheritance of adult-type lactose malabsorption. Lancet ii, 823-826.

Schmidt, G. H., Winton, D. J. \& Ponder, B. A. (1988). Development of the pattern of cell renewal in the crypt-villus unit of chimaeric mouse small intestine. Development 103, 785-790.

St Johnston, R. D. \& Gelbart, W. M. (1987). Decapentaplegic transcripts are localized along the dorsalventral axis of the Drosophila embyro. EMBO Journal 6, 2785-2791.

Suh, E., Chen, L., Taylor, J. \& Traber, P. G. (1994). A homeodomain protein related to caudal regulates intestine-specific gene transcription. Molecular and Cellular Biology 14, 7340-7351.

Traber, P. G., Wu, G. D. \& Wang, W. (1992). Novel DNA-binding proteins regulate intestine-specific transcription of the sucrase-isomaltase gene. Molecular and Cellular Biology 12, 3614-3627.

Tremml, G. \& Bienz, M. (1989). Homeotic gene expression in the visceral mesoderm of Drosophila embryos. EMBO Journal 8, 2677-2685.

Troelsen, J. T., Mehlum, A., OIsen, J., Spodsberg, N., Hansen, G. H., Prydz, H., Noren, O. \& Sjostrom, H. (1994). $1 \mathrm{~kb}$ of the lactase-phlorizin hydrolase promoter directs post-weaning decline and small intestinalspecific expression in transgenic mice. FEBS Letters 342, 291-296.

Wu, G. D., Wang, W. \& Traber, P. G. (1992). Isolation and characterization of the human sucrase-isomaltase gene and demonstration of intestine-specific transcriptional elements. Journal of Biological Chemistry 267, 7863-7870. 